\title{
A New Optimization Selection for Test Process of Equipment Manufacturing Based on Fused Algorithm
}

\author{
Gang LIU ${ }^{\mathrm{a}}$, Fang $\mathrm{LI}^{\mathrm{b}}$ \\ Department of Management Engineering, Naval University of Engineering \\ aemail:xinyi1229@163.com, bemaillifang600@126.com
}

Keywords: Optimization Selection; Genetic Algorithm; BP Neural Network; Simulated Annealing Algorithm

\begin{abstract}
Test optimization selection is a set cover problem, and heuristic algorithm for set covering problem is effective method. A genetic simulated annealing neural network fused algorithm was proposed by fusing the genetic algorithm, BP neural network and the simulated annealing algorithm, the genetic algorithm global search ability, strong ability of BP neural network training algorithm and fast search ability of simulated annealing algorithm were made full use of in this algorithm, the phenomenon falling into local optimum was avoided, and also the search efficiency and accuracy wad improved, the algorithm is applied to solve the test optimization selection problem. Example proves that this algorithm can effectively and quickly obtain test the optimal solution of optimization problems.
\end{abstract}

\section{Introduction}

In order to optimize the selection, the optimal combination test is chose in all possible combination of tests in the system, and the testability index can be met, while the minimum cost, include test time and test expense[1][2]. Perspective of mathematics, optimization selection is a test set covering problem, but we know set cover problem is a NP problem, when the system is on a big scale, to obtain the optimal solution is very difficult[3][4]. At present there are many more effective to solve the set covering problem of heuristic algorithm, such as genetic algorithm (GA) and simulated annealing algorithm (SA), neural network (ANN), tabu search (TS), ant colony system (ACO), etc., but these algorithms have their own advantages and defects, the effect is not ideal to solve alone, to complement each other mutual fusion has become the focus of future research[5][6][7].

This article is based on this idea, BP neural network and simulated annealing algorithm and introduced on the basis of traditional genetic algorithm, and a genetic simulated annealing neural network fusion algorithm has been formed, optimization selection can be solved faster and more accurate.

\section{Fusion algorithm}

\section{Operation process of fusion algorithm}

The operation process of fusion algorithm is shown in Figure 1.

\section{Extreme value and dead zone analysis of algorithm}

In the process of solving the traditional heuristic algorithm, the maximum and minimum values appear easily. These extreme values are very close to the optimal value of algorithm, but the fusion algorithm is based on genetic algorithm, and the advantages of genetic algorithm is strong global search capability, which can avoid the occurrence of extreme value in the whole search space[8][9]. Therefore, the occurrence of extreme value can be avoided in the fusion algorithm can the occurrence of extreme value.

Similarly, the genetic algorithm is good at global search, which can search to every corner of the space in the process of search, and the dead zone can be avoided in the fusion algorithm.

Because of strong global search ability of the genetic algorithm, extreme value and dead zone can be avoided, but the disadvantage of slow speed is also caused in the genetic algorithm. Moreover, 
The BP neural network and simulated annealing algorithm are introduced in the fusion algorithm, the search speed can be improved, and the optimal solution can solved quickly and effectively[10][11].

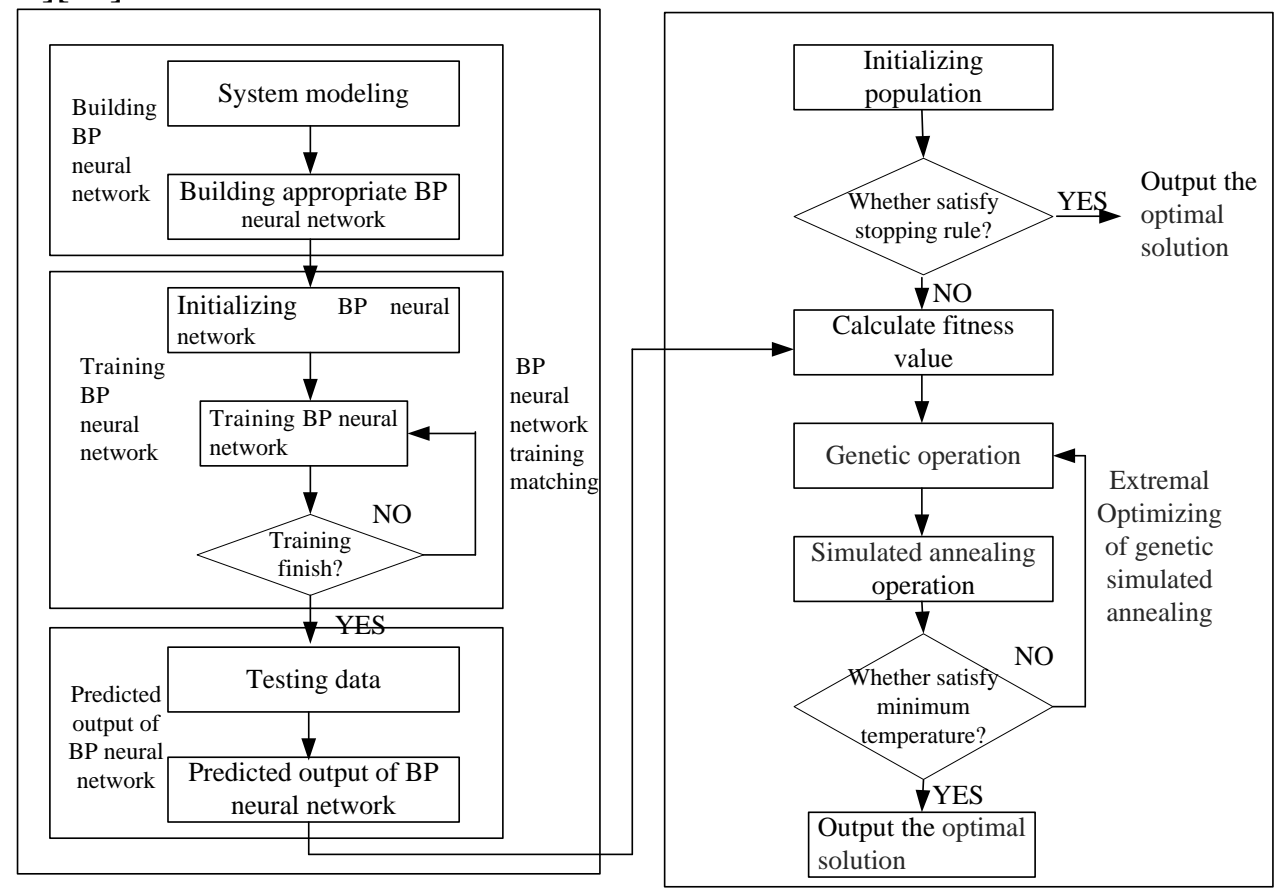

Fig.1. The operation process of fusion algorithm

\section{Simulation and verification}

In order to verify the validity of the algorithm, some system is chose for verification, the experiment is carried on the computer of P4 2.8 GMHZ CPU, 2GB memory, and programming algorithm is realized on the platform of Matlab7.0. Ten prior faults and fifteen alternative tests exist in this system, and the requested fault detection rate and fault isolation rate are 95\%. The corresponding relation between faults is shown in Table 1. The test cost (time and expense) is shown in Table 2.

Tab.1 Fault and test information correspondence table

\begin{tabular}{cccccccccccccccc}
\hline Fault & $T_{1}$ & $T_{2}$ & $T_{3}$ & $T_{4}$ & $T_{5}$ & $T_{6}$ & $T_{7}$ & $T_{8}$ & $T_{9}$ & $T_{10}$ & $T_{11}$ & $T_{12}$ & $T_{13}$ & $T_{14}$ & $T_{15}$ \\
\hline$S_{1}$ & 0 & 0 & 0 & 1 & 0 & 0 & 0 & 1 & 0 & 1 & 1 & 1 & 1 & 0 & 0 \\
$S_{2}$ & 0 & 0 & 1 & 0 & 1 & 0 & 0 & 0 & 0 & 0 & 1 & 1 & 0 & 1 & 0 \\
$S_{3}$ & 0 & 0 & 0 & 0 & 1 & 1 & 1 & 0 & 1 & 1 & 1 & 1 & 0 & 0 & 1 \\
$S_{4}$ & 0 & 1 & 0 & 0 & 0 & 1 & 1 & 0 & 0 & 0 & 0 & 0 & 0 & 1 & 1 \\
$S_{5}$ & 0 & 1 & 0 & 1 & 0 & 1 & 1 & 1 & 1 & 1 & 0 & 0 & 1 & 1 & 0 \\
$S_{6}$ & 0 & 0 & 0 & 1 & 1 & 0 & 0 & 1 & 1 & 1 & 0 & 1 & 1 & 1 & 1 \\
$S_{7}$ & 1 & 0 & 0 & 1 & 1 & 0 & 0 & 1 & 0 & 0 & 1 & 0 & 1 & 0 & 1 \\
$S_{8}$ & 1 & 1 & 1 & 0 & 0 & 1 & 1 & 0 & 1 & 1 & 1 & 0 & 0 & 1 & 0 \\
$S_{9}$ & 1 & 0 & 0 & 1 & 1 & 0 & 0 & 0 & 0 & 0 & 0 & 1 & 0 & 1 & 1 \\
$S_{10}$ & 1 & 1 & 1 & 1 & 0 & 0 & 1 & 0 & 0 & 0 & 1 & 0 & 1 & 1 & 0 \\
\hline
\end{tabular}

Tab.2 Test time and expense table

\begin{tabular}{cccccccccccccccc}
\hline Cost & $T_{1}$ & $T_{2}$ & $T_{3}$ & $T_{4}$ & $T_{5}$ & $T_{6}$ & $T_{7}$ & $T_{8}$ & $T_{9}$ & $T_{10}$ & $T_{11}$ & $T_{12}$ & $T_{13}$ & $T_{14}$ & $T_{15}$ \\
\hline Time & 2 & 1 & 3 & 5 & 1 & 3 & 3 & 2 & 1 & 3 & 4 & 5 & 1 & 2 & 2 \\
Expense & 300 & 200 & 100 & 100 & 100 & 200 & 300 & 100 & 200 & 200 & 100 & 400 & 100 & 100 & 200 \\
\hline
\end{tabular}

Assuming for different faults, the time and expense of the same test is the same, both time and expense per unit time and unit expenses. Parameters of algorithm are set as follows: the population 
size $M=20$, maximum evolution algebra $G=100$, crossover probability $p_{s}=0.4$, mutation probability $p_{c}=0.2,4000$ groups of input data of the function is taken, where 3900 groups of data are selected randomly for training network, 100 groups of data are selected randomly for testing network performance, predict output and the expected output contrast of BP neural network is shown in Figure 2, the best individual changes of fitness in the process of optimization is shown in Figure 3.

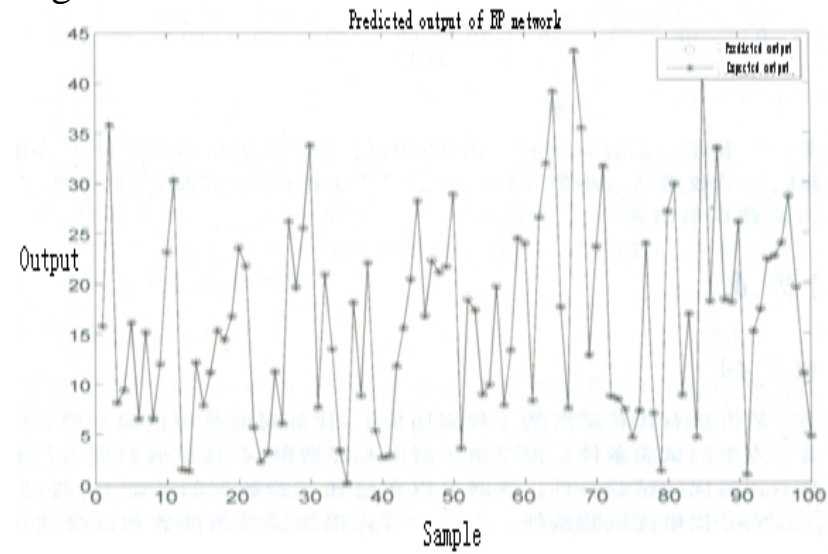

Fig.2. Predicted output of network

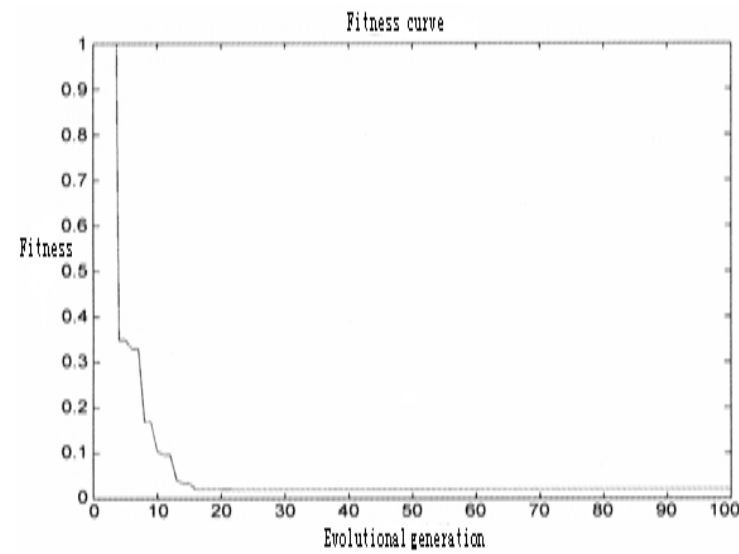

Fig.3. Fitness curve

The optimal solution can be solved by traditional genetic algorithm and fusion algorithm seen from the above table, but fewer iterations and fast solving speed in the fusion algorithm. Moreover, the solving speed of simulated annealing algorithm is faster than traditional genetic algorithm, but the solved result is worse than the optimal result.

In order to analyze the effect of solving general optimization selection problem by this algorithm, various faults - test are analyzed, and the fault test cost is identical, the coverage of fault test generated randomly, the calculated result is shown in Table 4.

Tab.3 Results contrast of each algorithm

\begin{tabular}{cccc}
\hline Algorithm & Optimal test set & $\begin{array}{l}\text { Test } \\
\text { cost }\end{array}$ & Iterations \\
\hline $\begin{array}{c}\text { Traditional } \\
\text { genetic }\end{array}$ & $T_{5}, T_{13}, T_{14}$ & 4.9 & 140 \\
$\begin{array}{l}\text { algorithm } \\
\text { Simulated } \\
\text { annealing } \\
\text { algorithm } \\
\text { Fusion }\end{array}$ & $T_{2}, T_{5}, T_{8}, T_{13}$ & 5.0 & 50 \\
algorithm & $T_{5}, T_{13}, T_{14}$ & 4.9 & \\
\hline
\end{tabular}

Tab.4 Results contrast

\begin{tabular}{ccccc}
\hline \multicolumn{3}{c}{ Problem parameter } & \multicolumn{2}{c}{ Results contrast } \\
\hline $\begin{array}{l}\text { Candidate } \\
\text { test } \\
\text { points }\end{array}$ & Faults & $\begin{array}{l}\text { Selective } \\
\text { test } \\
\text { points }\end{array}$ & Iterations & Time(s) \\
\hline 100 & 10 & 2 & 105 & 0.2563 \\
100 & 10 & 4 & 182 & 0.3568 \\
100 & 20 & 2 & 162 & 0.3639 \\
100 & 20 & 4 & 146 & 0.3258 \\
100 & 40 & 2 & 189 & 0.6938 \\
100 & 40 & 4 & 175 & 0.6783 \\
\hline
\end{tabular}

The results show that the effect solving the general problem by fusion algorithm is good, the numbers of iterations is less than 200 times, and the actual time is within 1s usually.

\section{Conclusions}

As systems become more and more complex, the relationship between fault and test also becomes more and more complex, it is necessary for optimization choice in the process of testability design. Fusion algorithm proposed in this paper, combining the advantages of genetic algorithm, BP neural network and simulated annealing algorithm, local optimal solution is avoided, search velocity is also improved, moreover, extreme value and dead zone analyzed.

The results show that the optimal solution can be quickly solved by the proposed fusion algorithm in the optimization selection problem, through the analysis of examples and general problem of optimal selection, and the requirements of the testability index can be satisfied, which has a certain guiding significance for system designers. 


\section{Acknowledgement}

In this paper, the research was sponsored by Natural Science Foundation of Naval University of Engineering (Project No. HGDQNEQJJ13016).

\section{References}

[1] LIU Gang, LI Fang, HU Bin. Test and modeling of vessel equipment based on relevant model[J]. Journal of Naval University of Engineering, 2012,24(8):46-51.

[2] Fang Li, Gang Liu, Peng Di. Research on relevance modeling method of vessel equipment testability[C]// 2012 International conference on Applied Mechanics and Manufacturing System, 2012:372-380.

[3] Gang Liu, Fang Li. A new testability optimization allocation approach[C]// $3^{\text {rd }}$ International Conference on Mechanical Science and Engineering, 2013:444-449.

[4] Gang Liu, Fang Li. A new sample selection method for testability verification experiment[C]// 3rd International Conference on Machine Design and Manufacturing Engineering, 2014:95-98.

[5] SU Yong-ding, QIAN Yan-ling, QIU Jing. Heuristic search in test selection[J]. CHINA MEASUREMENT TECHNOLOGY, 2005,31(5):46-49.

[6] ZHANG Yan-sheng, HUANG Kao-li, LIAN Guang-yao. Research on Selection of Testability Parameters of Missile Based on Modified AHP [J]. Computer Measurement \& Control, 2011,19(2): 412-414.

[7] WU Tao, YE Xiao-hui, WANG Hong-xia. Research on Problem of Test Selection Based on Quantum Genetic Algorithm [J]. Computer Measurement \& Control, 2010,18(11):2508-2510.

[8] AMONCHANCHAIGUL T, KREESURADEJ W. Input selection using binary particle swarm optimization[C]. International Conference on Computational Intelligence for Modeling Control and Automation, and International Conference on Intelligent Agents, Web Technologies and Internet Conference(CIMCA-IAWTIC’06), 2006.

[9] SADRI J, SUEN C Y. A genetic binary particle swarm optimization[C]. 2006 IEEE Congress on Evolutionary Computation Sheraton Vancouver Wall Centre Hotel, Vancouver, BC, Canada, July 16-21, 2006:656-663.

[10]AFSHINMANESH F, MARANDI A, RAHIMI-KIAN A. A novel binary particle swarm optimization method using system[C]. EUROCON 2005: Serbia \& November 22-24, 2005:217-220.

[11]Chen Xi-xiang,Qiu Jing,Liu Guan-jun. Optimal test selection based on hybrid BPSO and GA [J]. Chinese Journal of Scientific Instrument, 2009, 30(8):1674-1683. 